\title{
Características morfológicas de las médulas óseas en pacientes con infección por SARS- Cov2 del Hospital 2 de Mayo, Perú
}

\section{Morphological characteristics of bone marrows in patients with SARS-CoV-2 infection from Hospital 2 de Mayo, Peru}

Rafael Pichardo-Rodríguez ${ }^{1,2}$, Willy Peña-Oscuvilca ${ }^{3, b}$, David Díaz-Robles, ${ }^{1, b}$, Dennise MendozaSánchez ${ }^{1, \mathrm{~b}}$, Carlos Carrasco-Vergaray ${ }^{3, \mathrm{~b}}$, Herney Andrés García-Perdomo ${ }^{4, c}$, Oscar Ruíz-Franco ${ }^{1,3, \mathrm{~d}}$

\section{RESUMEN}

Introducción: A nivel medular, el SARS-CoV2 puede comprometer la hematopoyesis, manifestándose con citopenias y solo se cuenta con estudios realizados en autopsias. Objetivo: describir las características morfológicas de las médulas óseas de los pacientes hospitalizados por neumonía por COVID-19 en el Hospital Nacional "Dos de Mayo". El estudio: Estudio transversal retrospectivo llevado a cabo en pacientes con diagnóstico de COVID-19. Las lecturas de las médulas óseas fueron confirmadas por un hematólogo entrenado. Se utilizó estadística descriptiva para las variables cuantitativa y cualitativas. Hallazgos: Se incluyeron 30 pacientes. Los hallazgos más frecuentes fueron: macrófagos con citofagocitosis (87\%), hiperplasia con detención en la maduración de los progenitores mieloides en el (70\%). El 87\% de las muestras presentó eosinofilia. En el $57 \%$ de las muestras se observó ninguna o poca evidencia de formación plaquetaria. En el $40 \%$ se encontró $6 \%$ de células plasmáticas. Conclusión: La infección por SARS-CoV2 puede generar alteraciones medulares.

Palabras Clave: COVID-19; Médula Ósea; Hematopoyesis; Estudios Transversales (Fuente: DeCS-BIREME).

\section{ABSTRACT}

Background: At the spinal level, SARS-CoV2 can compromise hematopoiesis, manifesting itself with cytopenias and there are only studies carried out in autopsies. Objective: to describe the morphological characteristics of the bone marrow of patients hospitalized for pneumonia due to COVID-19 at the Hospital Nacional "Dos de Mayo". The study: Retrospective cross-sectional study carried out in patients diagnosed with COVID-19. The bone marrow readings were confirmed by a trained hematologist. Descriptive statistics were used for the quantitative and qualitative variables. Findings: 30 patients were included. The most frequent findings were: macrophages with cytophagocytosis (87\%), hyperplasia with arrest in the maturation of myeloid progenitors $(70 \%) .87 \%$ of the samples presented eosinophilia. Little or no evidence of platelet formation was observed in $57 \%$ of the samples. In $40 \%, 6 \%$ of plasma cells were found. Conclusion: SARS-CoV2 infection can generate spinal cord disorders.

Keywords: COVID-19; Bone Marrow; Hematopoiesis; CrossSectional Studies (Source: DeCS-BIREME).

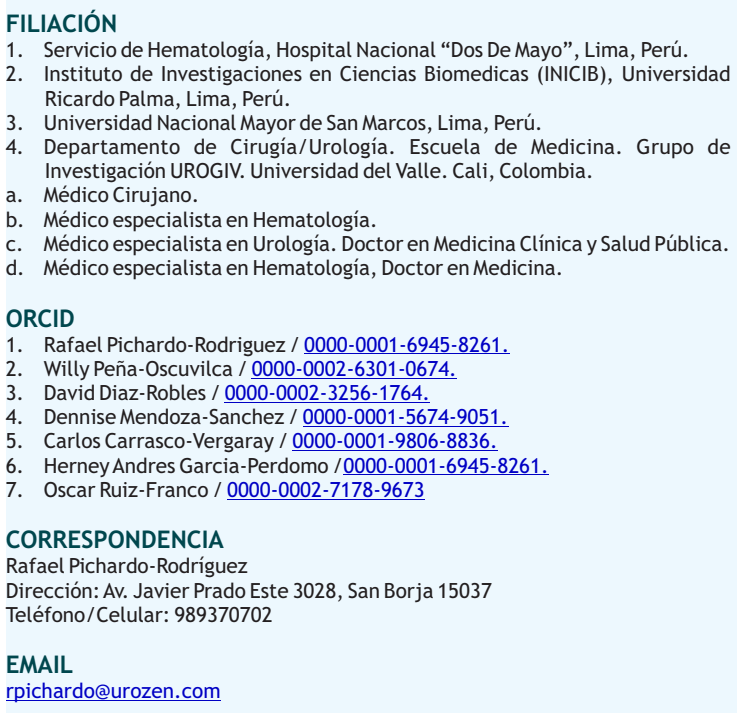

1. Servicio de Hematología, Hospital Nacional “Dos De Mayo”, Lima, Perú.

2. Instituto de Investigaciones en Ciencias Biomedicas (INICIB), Universidad Ricardo Palma, Lima, Perú.

. Universidad Nacional Mayor de San Marcos, Lima, Perú.

. Departamento de Cirugía/Urología. Escuela de Medicina. Grupo de Investigación UROGIV. Universidad del Valle. Cali, Colombia.

Médico Cirujano.

b. Médico especialista en Hematología.

Médico especialista en Urología. Doctor en Medicina Clínica y Salud Pública.

Médico especialista en Hematología, Doctor en Medicina.

ORCID

1. Rafael Pichardo-Rodriguez / 0000-0001-6945-8261.

2. Willy Peña-Oscuvilca / 0000-0002-6301-0674.

3. David Diaz-Robles / 0000-0002-3256-1764.

4. Dennise Mendoza-Sanchez / 0000-0001-5674-9051.

5. Carlos Carrasco-Vergaray / 0000-0001-9806-8836.

6. Herney Andres Garcia-Perdomo /0000-0001-6945-8261.

7. Oscar Ruiz-Franco / 0000-0002-7178-9673

CORRESPONDENCIA

Rafael Pichardo-Rodríguez

Dirección: Av. Javier Prado Este 3028, San Borja 15037

Teléfono/Celular: 989370702

EMAIL

rpichardo@urozen.com

CONFLICTOS DE INTERÉS

Los autores no declaran presentar conflictos de interés.

\section{FINANCIAMIENTO}

La presente investigación no ha recibido ninguna beca específica de agencias de los sectores público, comercial, o sin ánimo de lucro. Todo el proceso fue autofinanciado.

\section{REVISIÓN DE PARES}

Recibido: $17 / 06 / 2021$

Aceptado: 30/08/2021

\section{COMO CITAR}

Pichardo-Rodríguez, R. Peña-Oscuvilca, W., Diaz-Robles, D., MendozaSánchez, D., Carrasco-Vergaray, C., García-Perdomo, H., \& Ruiz-Franco, O. (2021). Características morfológicas de las médulas óseas en pacientes con infección por Sars-Cov2 del Hospital 2 de Mayo, Perú. Revista Del Cuerpo Médico Hospital Nacional Almanzor Aguinaga Asenjo, 14(3), 344 - 348. https://doi.org/10.35434/rcmhnaaa.2021.143.1256 


\section{INTRODUCCIÓN}

La enfermedad por el nuevo coronavirus 2019 (COVID-2019) ha generado una crisis sanitaria mundial que ha puesto en un gran peligro a la salud pública internacional ${ }^{(1)}$. Hasta la fecha, la infección por el coronavirus de tipo 2 causante del síndrome respiratorio agudo severo (SARS-CoV-2) ${ }^{(1)}$ continúa propagándose y las cifras de nuevos infectados crecen cada día, pese a las medidas de aislamiento y cuarentenas establecidas a nivel mundial.

Si bien, el sistema respiratorio es el más afectado, también hay disfunción de otros sistemas, destacando el sistema cardiovascular, gastrointestinal, endotelial, hematopoyético, hemostático entre otros, donde este virus ha podido ser identificado ${ }^{(2,3)}$. Se postula que, a nivel de sangre periférica, las alteraciones posiblemente estén asociadas a un daño a nivel medular, debido a que en los extendidos de sangre periférica se halla linfocitos reactivos con características plasmocitoides, así como alteraciones en los granulocitos como: hipergranulación, hiposegmetación, hipersegmentación e hipercondensación nuclear ${ }^{(3)}$.

Concordante a ello, se ha descrito a nivel medular, que el virus puede comprometer la hematopoyesis, manifestándose con citopenias (trombocitopenia y linfopenia) y al estudio microscópico, la presencia de hematofagocitosis ${ }^{(4)}$. Cabe destacar, que gran parte de los estudios han sido realizados a partir de autopsias y no de pacientes en la fase aguda de la enfermedad ${ }^{(5)}$. En nuestra experiencia como Servicio de Hematología del Hospital Nacional "Dos de Mayo" (HNDM), hemos observado en las diferentes muestras de médulas óseas de pacientes con COVID-19, la presencia de alteraciones como: plasmocitosis reactiva, displasias, que son similares a aquellas encontradas en pacientes con infección por el virus de la inmunodeficiencia humana $(\mathrm{VIH})^{(6,7)}$. Debido a la ausencia de evidencia local y consistente a nivel internacional sumado al importante impacto del virus a nivel de la médula ósea y en la necesidad de generar evidencia para mejorar la toma de decisiones y generar estudios posteriores, consideramos relevante la ejecución del presente estudio.

El objetivo fue describir las características morfológicas de las médulas óseas de los pacientes hospitalizados por neumonía por COVID-19 en el HNDM.

\section{ELESTUDIO}

\section{Diseño de estudio}

Estudio observacional transversal retrospectivo teniendo en cuenta los lineamientos de la declaración STROBE ${ }^{(8)}$. El trabajo fue registrado en la base de datos para estudios observacionales PRISA del Instituto Nacional de Salud del Perú, contando con el siguiente código de registro: El00000001767. El estudio fue evaluado y aprobado por la Oficina de Apoyo a la Docencia e Investigación del HNDM con el número de registro 1085-2020.

\section{Contexto}

Se llevó a cabo en pacientes con diagnóstico de neumonía por COVID-19 atendidos en el HNDM entre los meses de octubre a diciembre del año 2020 que fueron sometidos a un aspirado de médula ósea para encontrar la causa de las alteraciones hematológicas observadas en sangre periférica.

\section{Participantes}

Se incluyeron a pacientes mayores de 18 años con diagnóstico confirmado de neumonía por COVID-19 mediante prueba rápida o molecular que presentaban alteraciones en hemograma y frotis de lámina periférica y se excluyeron a aquellos con historia clínica incompleta, ilegible o extraviada y neumonía bacteriana y/o fúngica. Los pacientes se encontraban inscritos en el libro de registro de las salas de hospitalización COVID-19, que se utilizó como fuente para el marco muestral y selección de muestra.

\section{Variables}

La característica morfológica medular se definió como la descripción de la médula ósea (tinción Wright y Azul de Prusia, también llamada coloración de Perls para la hemosiderina) vista bajo microscopio reportado por un hematólogo entrenado. Esta estuvo compuesta de los siguientes parámetros:

Hemosiderina: Cuantificado en cruces $(+)$, siendo 0 la ausencia de hemosiderina y 4+ la concentración máxima.

- Celularidad: Cuantificado en +, siendo 0 la ausencia de células hematopoyéticas y 4+ como la celularidad máxima.

- Relación mielo/eritroide: Cuantificado como la relación entre la celularidad de la línea mieloide y eritroide. Se considera normal cuando la relación es de $3 / 1$. Y alterada cuando se pierde esta relación.

- Serie eritroide: Descripción de las características morfológicas y cuantitativas (hiperplasia eritroide: relación mielo/eritroide $<3 / 1$; hipoplasia eritroide: relación mielo/eritroide $>3 / 1$ ) de las células hematopoyéticas de la línea eritroide.

- Serie mieloide: Descripción de las características morfológicas y cuantitativas de las células hematopoyéticas de la línea mieloide.

- Serie megacariocítica: Descripción de las características morfológicas y cuantitativas (hiperplasia megacariocítica: >10 megacariocitos por espícula de tejido medular; hipoplasia megacariocítica: $<5$ megacariocitos por espícula de tejido medular) de las células hematopoyéticas de la línea megacariocítica.

- Células plasmáticas: Reporte de porcentaje y morfología. Se consideró normal hasta un recuento del $5 \%$.

Se midieron variables adicionales como sexo, edad, comorbilidades, tiempo de enfermedad, saturación de oxígeno, síntomas, analíticas de laboratorio (hemoglobina, leucocitos, plaquetas, linfocitos, Dímero D, Lactato Deshidrogenasa, Ferritina, Fibrinógeno y Troponina) y muerte.

\section{Fuente de datos}

Los datos fueron extraídos de las historias clínicas de los pacientes seleccionados y registrados en un instrumento estandarizado elaborado en base a los objetivos del estudio. 


\section{Sesgos}

Se midieron variables objetivas con un instrumento estandarizado y los informes de las lecturas de las médulas óseas fueron corroboradas por un hematólogo entrenado (ORF) para evitar los sesgos de medición.

\section{Tamaño de muestra}

Debido a la baja frecuencia de aspirado medular en pacientes con neumonía por COVID-19 se incluyeron a todos los pacientes encontrados.

\section{Análisis estadístico}

Se presentaron frecuencias y porcentajes para las variables cualitativas, así como medias y desviación estándar para las variables cuantitativas en función a las pruebas de normalidad (Shapiro-Wilk, se consideró significativo a un valor de $P>0,05)$. Los datos fueron procesados en el lenguaje de programación estadística $\mathrm{R}$ ( $\mathrm{R}$ Foundation for Statistical Computing, Vienna, Austria) en la interfaz gráfica JAMOVI versión 1.8.1.

\section{HALLAZGOS}

Se incluyeron a 30 pacientes que cumplieron con los criterios de selección. El sexo más frecuente fue el masculino (77\%;

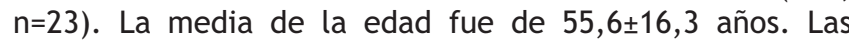
comorbilidades más frecuentes fueron obesidad e hipertensión arterial $(23 \%, n=7$ y $27 \%, n=8)$. El promedio de días desde el ingreso a hospitalización hasta la toma del aspirado medular fue de 10 días. Las características generales de los pacientes se presentan en la tabla 1.

Tabla 1. Características generales de los pacientes.

\begin{tabular}{lccc} 
& $\mathrm{N}$ & Femenino $(\mathrm{n}=7)$ & Masculino $(\mathrm{n}=23)$ \\
Edad & 30 & $50 \pm 23,1$ & $57,4 \pm 13,9$ \\
Hipertensión arterial & 30 & $21,7 \%(2)$ & $28,6 \%(5)$ \\
Diabetes mellitus tipo 2 & 30 & $28,6 \%(2)$ & $8,7 \%(2)$ \\
Obesidad & 30 & $28,6 \%(2)$ & $26,1 \%(6)$ \\
Sobrepeso & 30 & $28,6 \%(2)$ & $13 \%(3)$ \\
Enfermedad renal crónica & 30 & $14,3 \%(1)$ & $8,7 \%(2)$ \\
Otra comorbilidad & 30 & $14,3 \%(1)$ & $8,7 \%(2)$ \\
Tiempo de enfermedad (días) & 30 & $6,5 \pm 1,9$ & $15,1 \pm 17,8$ \\
Sensación de alza térmica & 30 & $78,3 \%(5)$ & $71,4 \%(17)$ \\
Tos & 30 & $100 \%(7)$ & $60,9 \%(14)$ \\
Cefalea & 30 & $28,6 \%(2)$ & $21,7 \%(5)$ \\
Disnea & 30 & $71 \%(5)$ & $69,6 \%(16)$ \\
Malestar general & 30 & $57 \%(4)$ & $52,2 \%(12)$ \\
Otros síntomas & 30 & $28,6 \%(2)$ & $30 \%(7)$ \\
Saturación de oxígeno $(\%)$ & 30 & $94,3 \pm 4,9$ & $92,8 \pm 4,1$ \\
Hemoglobina $(\mathrm{g} / \mathrm{dL})$ & 30 & $11,2 \pm 1,8$ & $13 \pm 1,8$ \\
Plaquetas (plaquetas/mm $\left.{ }^{3}\right)$ & 30 & $373857 \pm 194385$ & $310304 \pm 155262$ \\
Linfocitos (linfocitos/mm $\left.{ }^{3}\right)$ & 30 & $1207 \pm 561$ & $946 \pm 503$ \\
Dímero D (mg/dL) & 30 & $1,2 \pm 0,9$ & $5,7 \pm 1,5$ \\
Lactato deshidrogenasa $(\mathrm{UI} / \mathrm{L})$ & 30 & $274 \pm 103$ & $437 \pm 206$ \\
Ferritina (mg/dL) & 30 & $448 \pm 187$ & $706 \pm 443$ \\
Fibrinógeno $(\mathrm{mg} / \mathrm{dL})$ & 30 & $592 \pm 107$ & $807 \pm 260$ \\
Troponina (ng/dL) & 30 & $5,5 \pm 2$ & $5,7 \pm 8,2$ \\
\hline
\end{tabular}

Del total de muestras de médula ósea, el 46,3\% ( $n=14)$, presentó hemosiderina en 3-4+. Sin embargo, en el $26,7 \%$ $(n=8)$ estuvo ausente con un dosaje promedio de ferritina de $702 \pm 306 \mathrm{mg} / \mathrm{dL}$. Por otro lado, en el $63,3 \%(\mathrm{n}=19)$, la celularidad estuvo en $4+$. En cuanto a la relación mielo/eritroide más frecuente fue de $4 / 1$, representando el $63,3 \%(n=19)$.

A nivel de la serie eritroide, en el $30 \% \quad(n=9)$ de las características fueron normocelulares (sin alteración patológica de los precursores de la serie eritroide) predominando los ortrocromáticos. En un 26,6\% ( $n=8)$ se observó la detención de la maduración (maduración megaloblástica). Y se objetivó un solo caso $(3,3 \%)$ de hipoplasia donde predominó los eritroblastos.

A nivel de la serie mieloide, en el $87 \%(n=26)$ se observó macrófagos con citofagocitosis, y en el $70 \% \quad(n=21)$ se encontró hiperplasia con detención en la maduración de los progenitores mieloides. El $87 \%(\mathrm{n}=26)$ de las muestras presentó eosinofilia (incremento del número de eosinófilos a nivel de la médula ósea). Se observó detección en la maduración en el $17 \%(n=5)$. Solo se objetivaron 3 casos de dismielopoyesis $(9,9 \%)$ caracterizados por la presencia de neutrófilos con hiposegmentación nuclear e hipogranulación a nivel citoplasmático.

En la serie megacariocítica, se observó en el 13,2\% ( $n=4)$ hiperplasia. Por otro lado, en el $57 \%(n=17)$ de las muestras se observó poca o nula formación de plaquetas por parte de los megacariocitos.

En todas las muestras se encontraron células plasmáticas maduras. En el $40 \%(n=12)$ se encontró $6 \%$ de células plasmáticas. El máximo porcentaje de células plasmáticas en las muestras fue de $8 \%(6,7 \%, n=2)$ y el menor del $3 \%(33 \%$, $n=10)$. En la figura 1 se presentan los cambios morfológicos encontrados en nuestro estudio.
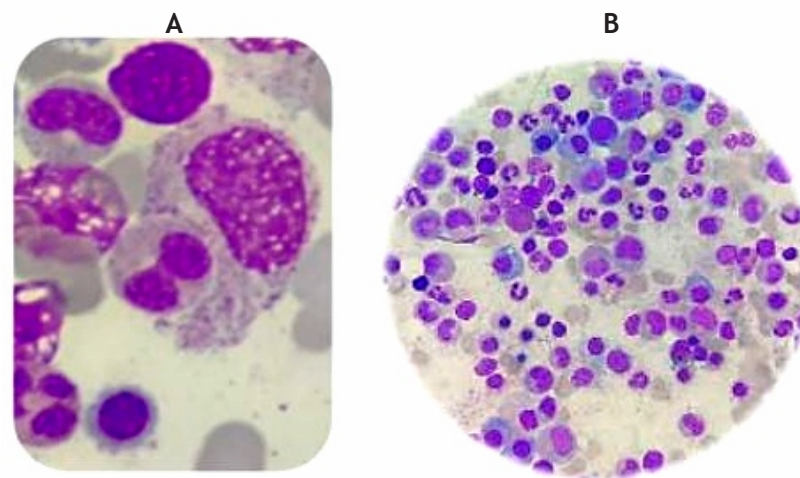

Figura 1.

Características morfológicas de las médulas óseas encontradas en nuestros pacientes. A. Se observa el proceso de citofagocitosis (macrófago con núcleo oval y cromatina irregular fagocitando a un abastonado). B. encontramos una médula ósea hipercelular en la serie mieloide, además de incremento de células plasmáticas maduras (Aumento: 100X; Coloración Wright

\section{DISCUSIÓN}

La infección por el SARS-COV-2 ha demostrado ser una enfermedad multisistémica, afectando a diversos órganos pese a que el principal objetivo del virus es el aparato respiratorio. Además se puede encontrar un daño en los progenitores hematopoyéticos, manifestándose clínicamente como cambios en sangre periférica, así 
Revista del Cuerpo Médico del HNAAA, Vol 14 (3) - 2021

reportado en diversos estudios ${ }^{(9,10)}$. Incluso se ha publicado la asociación de pancitopenia a la infección por SARS-COV-2, que se resolvió espontáneamente al controlarse el cuadro ${ }^{(11)}$.

Se ha descubierto que el receptor de la enzima convertidora de la angiotensina 2 se expresa en la médula ósea, incluyendo a las células madres hematopoyéticas, abriendo la posibilidad de un mecanismo fisiopatológico para poder explicar este fenómeno ${ }^{(12)}$. Por otro lado, también se ha descrito que el inflamosoma de la proteína 3 receptora similar al dominio de unión a nucleótidos expresado en las células madre hematopoyéticas podría exacerbar la tormenta de citocinas ${ }^{(13)}$. Aún hay mucho por conocer y las publicaciones relacionadas al daño de la médula ósea por el COVID-19 son todavía escasas.

En nuestro estudio encontramos que la celularidad más frecuente fue de 4+ al igual que la relación mielo/eritroide de 4/1. Esto hace referencia a la presencia de hiperplasia de los precursores hematopoyéticos, concordante con la hiperplasia con detención en la maduración objetivada en los precursores mieloides. De manera similar, Swoboda et al, reporta un incremento sustancial de los precursores mieloides con predominio de mielocitos y metamielocitos ${ }^{(5)}$. De la misma forma, también es compatible con los hallazgos medulares reportados por Amy et al, encontrados en la autopsia de siete pacientes fallecidos por COVID-19, reportándose una médula ósea hipercelular con un aumento de los megacariocitos que demostraba un agrupamiento focal, además, hubo maduración progresiva de las series mieloides con desplazamiento a la izquierda y eritropoyesis reducida. Se conservó en todo momento la presencia de las tres líneas celulares en las muestras de médulas óseas de manera similar a lo descrito por Swoboda et $\mathrm{al}^{\left({ }^{(5)}\right.}$.

Otro hallazgo reportado en la literatura es la presencia de hemofagocitosis como se observó en los aspirados de médula ósea de tres pacientes con COVID-19 grave $^{(12)}$. De igual manera, en otro estudio también se describió la presencia de macrófagos con hemofagocitosis en la médula ósea en pacientes con COVID-19. En nuestros pacientes encontramos que el $70 \%$ presentaban dicha alteración. Adicionalmente, otra alteración reportada, es la presencia de células plasmáticas incrementadas en médula ósea. En nuestros pacientes encontramos hiperplasia en el $40 \%$ de las muestras, siendo el conteo más frecuente un $6 \%$ de la celularidad medular.

En el estudio realizado por Amy et al, encontraron un mayor número de megacariocitos en la médula ósea, y la morfología apuntaba a la producción activa de plaquetas. En nuestros pacientes, encontramos una ausencia o reducción en la formación plaquetaria en el $57 \%$ y solo el $13,2 \%$ presentó hiperplasia megacariocítica. Por otro lado, se ha reportado la identificación de viriones en los megacariocitos mediante microscopía electrónica ${ }^{(12)}$. Nosotros no pudimos confirmar su presencia, debido a que no contamos con dicha tecnología en nuestro hospital.

Algo resaltante ha sido encontrar eosinofilia en el $87 \%$ de las muestras. Se reporta que, en los pacientes infectados, la prevalencia de eosinofilia es alta $(28,7 \%)$, sin embargo, este recuento es solo a nivel de sangre periférica y que probablemente la eosinofilia se asocie a un menor riesgo de complicaciones e ingreso a la unidad de cuidados intensivos ${ }^{(14)}$. Por otro lado, la eosinopenia se ha asociado a un peor pronóstico ${ }^{(15)}$. Es necesario evaluar en futuro la asociación de este hallazgo medular al pronóstico de los pacientes.

No podemos asumir que la ausencia de hemosiderina haya sido secundaria a la infección, debido a que la instauración de una deficiencia de hierro conlleva un tiempo de latencia prolongado, además para poder originar una ferropenia marcada como la que se pudo observar en nuestros pacientes y con ello una anemia se debe de pasar por diversas fases ${ }^{(16)}$. Es probable que haya estado asociado a los hábitos nutricionales previos de los pacientes y/o a sus comorbilidades.

Las mujeres evidenciaron con mayor frecuencia comorbilidades y síntomas, sin embargo, la saturación de oxígeno promedio fue mayor en comparación a los varones. El Dímero D, el lactato deshidrogenasa, la ferritina, el fibrinógeno fueron en promedio mayores en los varones en comparación a las mujeres. El nivel de plaquetas y linfocitos fueron menores en los varones en relación a las mujeres. Estas alteraciones son similares a lo reportado en la literatura ${ }^{(17,18)}$.

Estos cambios medulares son similares a los que se observan en la infección por el $\mathrm{VIH}$, ya que los receptores del $\mathrm{VIH}$ también se expresan en las células madre hematopoyéticas ${ }^{(19)}$. Igualmente, se ha observado alteraciones hematológicas en sangre periférica en la infección por otros tipos de coronavirus como el coronavirus de tipo 1 causante del síndrome respiratorio agudo y el coronavirus del síndrome respiratorio de Oriente Medio severo, que probablemente tengan efectos similares en la médula ósea ${ }^{(12)}$. En nuestra experiencia como Servicio de Hematología, los hallazgos que hemos encontrado se nos hacen similares con las alteraciones vistas en los pacientes infectados con el VIH sin tratamiento antiviral de gran actividad. Probablemente el SARS-COV-2 y el VIH tengan mecanismos fisiopatológicos similares a nivel de los progenitores hematopoyéticos.

Esto nos permite comprender aún más la presencia de las alteraciones en sangre periféricas mencionadas en la literatura y vistas en nuestros pacientes, dándonos luces de nuevas alteraciones que no habían sido encontradas en otras poblaciones como la eosinofilia a nivel medular, que probablemente se asocie a una buena evolución de la enfermedad. La infección por SARS-COV-2 conlleva a alteraciones importantes en la médula ósea, y en nuestra población, se observa algunas particularidades en relación a los reportado en otros estudios. Siendo relevante la conducción de estudios prospectivos para evaluar la evolución del compromiso medular, y su asociación a eventos clínicos importantes como mortalidad.

\section{Fortalezas y limitaciones}

La principal fortaleza de nuestro estudio es que las alteraciones morfológicas encontradas reflejan los cambios que genera el virus in vivo, durante la fase aguda de la enfermedad, ayudándonos a comprender mejor sus efectos 
histopatológicos que deben corroborarse con estudios prospectivos de seguimiento. La debilidad más importante fue que al ser un estudio retrospectivo, se incurriría en un sesgo de medición al registrar los reportes de las médulas óseas realizada por otros hematólogos, sin embargo, estos resultados fueron corroborados por un médico hematólogo entrenado (ORF), tratando así de reducir este error sistemático. Una debilidad adicional fue que no pudimos corroborar la severidad de la infección en todos los pacientes por la falta de su registro en las historias clínicas, no obstante, los pacientes que son ingresados en nuestro hospital son casos moderados y severos, así que nuestros resultados solo podrían ser aplicados en esta población.

Los pacientes con infección por SARS-COV2, presentan alteraciones medulares entre los que destacan: la citofagocitosis con hiperplasia y detención de la maduración en la serie mieloide, poca o nula producción plaquetaria en los megacariocitos y eosinofilia. Se requieren estudios prospectivos que evalúen la evolución del compromiso medular posterior a la infección aguda y su relevancia pronóstica en relación a la severidad y mortalidad.

\section{REFERENCIAS BIBLIOGRÁFICAS}

1. Shi Y, Wang G, Cai X, Deng J, Zheng L, Zhu H, et al. An overview of COVID-19. J Zhejiang Univ Sci B. 2020; 21(5):343-60. doi:10.1631/jzus.B2000083.

2. Terpos E, Ntanasis-Stathopoulos I, Elalamy I, Kastritis E, Sergentanis TN, Politou $M$, et al. Hematological findings and complications of COVID-19. Am J Hematol. 2020; 95:834-47. doi:10.1002/ajh.25829.

3. Villa Palacio MI, López Henao E. Hematological Findings in COVID-19. Nova. 2020;18(spe35):75-9. doi:10.22490/24629448.4189.

4. Debliquis A, Harzallah I, Mootien JY, Poidevin A, Labro G, Mejri A, et al. Haemophagocytosis in bone marrow aspirates in patients with COVID-19. Br J Haematol. 2020;190(2):e70-3. doi:10.1111/bjh.16860

5. Swoboda J, Wittschieber D, Sanft J, Kleemann S, Elschner S, Ihle H, et al. Bone marrow haemophagocytosis indicates severe infection with severe acute respiratory syndrome coronavirus 2 . Histopathology. 2021;78(5):727-37. doi:10.1111/his.14281.

6. Dhurve SA, Dhurve AS. Bone Marrow Abnormalities in HIV Disease. Mediterr J Hematol Infect Dis. 2013;5(1):e2013033. doi:10.4084/MJHID.2013.033.
7. O'Brien T, Bowman L. Quantification of Marrow Plasmacytosis in HIV $\mathrm{P}$ a t i e n t s. Blood. $2015 ; 126(23): 4630$. doi:10.1182/blood.V126.23.4630.4630.

8. Vandenbroucke JP, Von Elm E, Altman DG, Gøtzsche PC, Mulrow CD, Pocock SJ, et al. Mejorar la comunicación de estudios observacionales en epidemiología (STROBE): explicación y elaboración. Gaceta Sanitaria. 2009;23(2):158.e1-28. doi:10.1016/j.gaceta.2008.12.001.

9. Asghar M, Hussain N, Shoaib H, Kim M, Lynch TJ. Hematological characteristics of patients in coronavirus 19 infection: a systematic review and meta-analysis. J Community Hosp Intern Med Perspect. 2020;10(6):508-13. doi:10.1080/20009666.2020.1808360.

10. Liao D, Zhou F, Luo L, Xu M, Wang H, Xia J, et al. Haematological characteristics and risk factors in the classification and prognosis evaluation of COVID-19: a retrospective cohort study. Lancet Haematol. 2020;7(9):e671-8. doi:10.1016/S2352-3026(20)30217-9.

11. Hersby DS, Do TH, Gang AO, Nielsen TH. COVID-19-associated pancytopenia can be self-limiting and does not necessarily warrant bone marrow biopsy for the purposes of SARS-CoV-2 diagnostics. Ann Oncol. 2021;32(1):121-3. doi:10.1016/j.annonc.2020.09.020.

12. Rahman A, Niloofa R, Jayarajah U, Mel SD, Abeysuriya V, Seneviratne SL. Hematological Abnormalities in COVID-19: A Narrative Review. Am J Trop Med Hyg. 2021;104(4):1188-201. doi:10.4269/ajtmh.20-1536.

13. Ratajczak MZ, Kucia M. SARS-CoV-2 infection and overactivation of Nlrp3 inflammasome as a trigger of cytokine "storm" and risk factor for damage of hematopoietic stem cells. Leukemia. 2020;34(7):1726-9. doi:10.1038/s41375-020-0887-9.

14. Nair AP, Soliman A, Masalamani MAA, Sanctis VD, Nashwan AJ, Sasi S, et al. Clinical Outcome of Eosinophilia in Patients with COVID-19: A Controlled Study : Eosinophilia and COVID -19. Acta Biomed. 2020;91(4):e2020165. doi:10.23750/abm.v91i4.10564.

15. Rosenberg HF, Foster PS. Eosinophils and COVID-19: diagnosis, prognosis, and vaccination strategies. Semin Immunopathol. 2021;43(3):383-92. doi:10.1007/s00281-021-00850-3.

16. Saito H. Metabolism of iron stores. Nagoya J Med Sci [Internet]. 2014 [cited 2021 apr 25];76(3-4):235-54. Available from: https://www.ncbi.nlm.nih.gov/pmc/articles/PMC4345694/.

17. Li X, Xu Z, Wang T, Xu X, Li H, Sun Q, et al. Clinical laboratory characteristics of severe patients with coronavirus disease 2019 (COVID-19): A systematic review and meta-analysis. Clin Epidemiol Glob Health. 2021;9:184-90. doi:10.1016/j.cegh.2020.08.012.

18. Pormohammad A, Ghorbani S, Baradaran B, Khatami A, J. Turner R, Mansournia MA, et al. Clinical characteristics, laboratory findings, radiographic signs and outcomes of 61,742 patients with confirmed COVID-19 infection: A systematic review and meta-analysis. Microb Pathog. 2020;147:104390. doi:10.1016/j.micpath.2020.104390.

19. Akkina R. New insights into HIV impact on hematopoiesis. Blood. 2013;122(13):2144-6. doi:10.1182/blood-2013-08-518274. 